\title{
Criterios para el uso e investigación de medicamentos off-label frente al covid-19 en Latinoamérica
}

\section{Criteria for the use and investigation of off-label drugs against covid-19 in Latin America}

\author{
Yeison Harvey Carlosama-Rosero ${ }^{1}$
}

\section{RESUMEN}

La pandemia por COVID-19 representa una de los retos más importantes en materia de salud pública. La ausencia de evidencia científica sólida y la severidad del cuadro clínico han justificado el uso compasivo de medicamentos off-label. No obstante, el uso indiscriminado de estos medicamentos, sin un fundamento sólido por parte de los médicos puede

\begin{abstract}
The COVID-19 pandemic represents one of the most important challenges in public health's history. Severity of the clinical picture and lack of supporting scientific evidence have led to compassionate use of off-label medications. Indiscriminate use of these medications, however, can be harmfulforpatients. The Latin American medical and scientific community is called upon to
\end{abstract}

Historial del artículo:

Fecha de recepción: 26/01/2021

Fecha de aprobación: 18/05/2021

1 Universidad Cooperativa de Colombia. Facultad de Medicina. Grupo Interdisciplinario de Investigación en Salud y Enfermedad. Pasto, Colombia.

Correspondencia: Yeison Carlosama Rosero. Dirección: Universidad Cooperativa de Colombia. Calle 18 \# 47-150 Torobajo. Pasto. Colombia. Teléfono: 3216730843. Correo electrónico: yeharca@hotmail.com

Como citar este artículo: Carlosama-Rosero Y. Criterios para el uso e investigación de medicamentos off-label frente al covid-19 en latino américa. Revista de la Facultad de Ciencias de la Salud de la Universidad del Cauca. 2021;23(2):39-46. https://doi.org/10.47373/ rfcs.2021.v232.1778 
representar un peligro para la salud de los pacientes. Por otra parte, la comunidad médica y científica de Latinoamérica está llamada a evaluar hipótesis de investigación que mitiguen el impacto de la pandemia en nuestras poblaciones.

Tomando como ejemplo pedagógico el uso de la ivermectina, se proponen criterios como la plausibilidad biológica, la evidencia metodológica y estadísticamente aceptable, una respuesta clínica favorable, un perfil farmacológico seguro, la factibilidad y el interés altruista como sustento para el empleo de medicamentos offlabel durante la pandemia. Estos criterios posibilitarían un uso racional de medicamentos y priorizar en el contexto de una investigación las opciones terapéuticas posiblemente más efectivas.

Palabras clave: Pedagogía, ivermectina, COVID-19, educación médica, off-label.

\section{INTRODUCCIÓN}

La comunidad científica mundial ha volcado su interés en un tratamiento que permita curar a pacientes con COVID-19 o cuando menos mitigar su impacto. Sin embargo, hasta el momento pocos de los medicamentos en estudio muestran una evidencia científica sólida y las mejores esperanzas están depositadas en el desarrollo de una vacuna o en la información derivada de ensayos internacionales como el SOLIDARITY, INSERM y DisCoVeRy (1-3).

La severidad del cuadro clínico en algunos pacientes y la falta de evidencia científica han motivado a la comunidad médica mundial a emplear medicamentos fuera de indicación (offlabel) con fines compasivos y con la finalidad de evitar un desenlace fatal (4). No obstante, el uso de estos medicamentos sin un criterio médico y científico puede no solo ser inefectivo sino también perjudicial, tal como parece haber ocurrido (según la información disponible hasta el momento) con la cloroquina y la hidroxicloroquina. En este sentido, los ensayos clínicos en proceso de revisión por pares así como el artículo publicado en la revista Lancet por Mandeep et al. muestran un panorama desalentador para estos medicamentos $(5,6)$. En particular, este estudio recoge información de más de 96.000 pacientes y emplea elementos metodológicos robustos que permiten confiar en la validez de sus conclusiones. Entre las conclusiones se citan un incremento significativo de la mortalidad y la aparición de arritmias cardiacas en pacientes tratados con cloroquina o hidroxicloroquina. evaluate research hypotheses of treatments that could mitigate the impact of the pandemic on our populations.

Taking the use of ivermectin as an example, various criteria such as biological plausibility, methodological and statistically acceptable evidence, a favorable clinical response, a safe pharmacological profile, feasibility, and altruistic interest are proposed to support the use of offlabel treatmentsduring the pandemic. These criteria would allow a more rational use of medications and prioritize research on potentially effective therapeutic options.

Keywords: Pedagogic, COVID-19, medical education, off-label.
En distintos medios de comunicación -en particular a través redes sociales-, se ha promocionado por parte de algunos médicos el empleo de múltiples terapias off-label, en su mayoría, sin el suficiente soporte científico y sustentadas en criterios subjetivos, basados esencialmente en observaciones anecdóticas en algunos pacientes. En el escenario de una pandemia como la actual donde la desinformación, el temor y el criterio poco estructurado han sido una constante, el empleo irracional de dichas terapias puede representar un peligro adicional para la salud de la población (7).

Por otra parte, la Organización Mundial de la Salud publicó el 15 de octubre de 2020 los resultados del ensayo Solidarity, un estudio internacional que evaluó el efecto de cuatro tratamientos (remdesivir, hidroxicloroquina, lopinavir/ ritonavir e interferón) concluyendo que los medicamentos en mención tienen poco o ningún efecto sobre la mortalidad, estancia hospitalaria o requerimiento de ventilación mecánica (8). Estos hallazgos sugieren que se deben explorar otras alternativas terapéuticas frente al COVID-19. Tradicionalmente, el uso de medicamentos se ha fundamentado en la mejor evidencia disponible, la cual a su vez deriva de estudios metodológicamente robustos donde se evalúa la efectividad y el perfil de seguridad del medicamento. Por esta razón, los niveles de evidencia causal son un referente innegable a la hora de decidir el uso de un medicamento (9).

Aun en el contexto de una pandemia se necesita evidencia científica sólida derivada preferiblemente de ensayos clínicos 
aleatorizados bien diseńados y con validez externa. No obstante, en una situación donde la salud y la vida de miles de personas está en riesgo, esperar los resultados de los ensayos clínicos puede parecer una estrategia que responda de manera satisfactoria a la dinámica de la pandemia. En este escenario, parece razonable explorar alternativas para el uso de medicamentos off-label a la vez que se ponen en marcha protocolos de investigación que generan evidencia de buena calidad. Esta exploración debe estar muy bien fundamentada en criterios razonablemente aceptables y sin exponer a un riesgo injustificado a los pacientes.

El papel de los investigadores y de las sociedades médicas de Latinoamérica no debe limitarse a esperar los resultados de investigaciones desarrolladas en Europa, Asia o Estados Unidos, donde las condiciones económicas y el acceso a los servicios de salud son distintos. Dados los limitados recursos para la investigación, es necesario priorizar las alternativas farmacológicas probablemente más efectivas, viables y seguras de desarrollar en nuestros contextos. Se ha sugerido que dicha priorización para el empleo de medicamentos off-label frente al COVID-19 se realice bajo estricta vigilancia médica, idealmente en el marco de protocolos de investigación juiciosos y bien regulados, y fundamentada en criterios bien definidos. Se proponen como criterios a considerar la plausibilidad biológica, existencia de evidencia metodológica y estadística aceptable, evidencia de una respuesta clínica favorable, un perfil farmacológico seguro, factibilidad e interés altruista. Estos aspectos se discutirán tomando como ejemplo didáctico algunos referentes bibliográficos relacionados con el uso de la ivermectina, medicamento de uso bastante controversial durante la pandemia y cuya calidad de evidencia frente al COVID por el momento es cuanto menos cuestionable e insuficiente.

\section{PLAUSIBILIDAD BIOLÓGICA}

Este criterio implica que el medicamento o la terapia propuesta sea biológicamente activa frente al SARS-CoV-2, por lo tanto, debe existir una base racional que permita sustentar su uso a través de fundamentos biomédicos conocidos.

En el caso de la ivermectina, distintos estudios preclínicos han demostrado actividad biológica frente a algunos virus como el Chikungunya, Alfa virus, Virus de la Encefalitis Equina Venezolana y distintos tipos de dengue $(10,11)$. Además, existe evidencia preclínica de su efectividad contra SARS-CoV-2. En el estudio de Caly y colaboradores se demostró que la adición de una sola dosis de ivermectina a cultivos Vero-hSlam redujo en 5000 veces el RNA viral después de 48 horas (12).
El conocimiento adquirido de la pandemia del SARS y MERS, ocasionadas por agentes microbiológicos que comparten notables similitudes con el SARS-CoV-2 (13) permiten plantear un posible efecto biológico de la ivermectina a través de un bloqueo de la replicación nuclear del virus y de la estimulación de la respuesta inmunológica del paciente. En este sentido, después de ingresar a la célula, las partículas virales se transfieren al núcleo a través de un heterodímero llamado importina (IMP) $\alpha / \beta 1$. Se ha planteado que la ivermectina impide la formación del heterodímero al unirse a la importina y bloqueando la translocación nuclear del virus (14).

Por otra parte, se ha demostrado en algunas infecciones por SARS coronavirus una respuesta tardía frente al interferón bloqueando respuestas inmunes como la diferenciación de linfocitos citotóxicos, activación de células dendríticas y natural killer, expresión de moléculas del complejo mayor de histocompatibilidad y producción de inmunoglobulina E $(15,16)$. La respuesta tardía al interferón se explica por un bloqueo de la vía de transcripción STAT-1 a través de una proteína viral llamada ORF-6. Esta proteína, secuestra la importina y las carioferinas en el aparato de Golgi o el retículo endoplásmico. Al estar secuestradas en las organelas celulares, las carioferinas no se translocan al núcleo y no activan genes respondedores frente al interferón (14). Se ha propuesto que la unión de la ivermectina con las importinas bloquea el secuestro en las organelas y facilita la transcripción nuclear de carioferinas que activan genes respondedores frente al interferón.

En resumen, la ivermectina tendría un efecto antiviral al bloquear la replicación nuclear del virus y al estimular la respuesta inmunológica a través del interferón, razón por la cual se puede concluir que existe una explicación plausible que ampara su potencial efectividad -al menos a nivel biológico-frente a la enfermedad.

\section{EVIDENCIA METODOLÓGICA Y ESTADÍSTICAMENTE ACEPTABLE}

En este criterio se propone que el medicamento o la terapia propuesta tenga o haya demostrado efectividad a través de estudios con un diseńo metodológico aceptable. Evidentemente, los estudios más robustos desde el punto de vista científico son los ensayos clínicos aleatorizados con cegamiento. No obstante, el diseńo y ejecución de estos tipos de estudios es bastante complejo, demandan una inversión importante de tiempo y en la actualidad gran parte de los ensayos clínicos se encuentran en fase de reclutamiento o ejecución por lo cual sus resultados no estarán disponibles en el corto plazo. En estas condiciones, 
deberán analizarse cuidadosamente los resultados de otros diseńos epidemiológicos. En orden de jerarquía causal deberán ponderarse primero los estudios analíticos que emplean un grupo control para establecer una medida de riesgo (Cohortes, seguidos por los estudios de casos y controles) y en última instancia los estudios descriptivos y series de casos (17). Los estudios analíticos se consideran más robustos dado que permiten hacer una comparación entre dos grupos presumiblemente muy similares excepto en relación a una variable de interés que se quiere evaluar. Estos estudios posibilitan establecer diferencias en la aparición de eventos entre los grupos, así como cuantificar la magnitud de tales diferencias (18). Para el caso de la terapia farmacológica esas diferencias pueden traducirse en un efecto clínicamente medible a través de pruebas estadísticas que permitan corroborar una hipótesis con cierto grado de certeza y anulando el potencial papel del azar (19).

En el caso de la Ivermectina resalta una publicación de la investigación de Rajter y colaboradores (20). Esta publicación corresponde a un estudio analítico observacional basado en registro clínicos que incluyó una población de 280 pacientes de cuatro centros hospitalarios de los Estados Unidos. En este estudio se evaluó el efecto del tratamiento con ivermectina versus tratamiento sin ivermectina. En el grupo que recibió ivermectina se incluyeron pacientes que recibieron al menos una dosis de ivermectina de $200 \mathrm{ug} / \mathrm{Kg}$ además del tratamiento convencional, documentándose una reducción significativa de la mortalidad ( $15 \%$ versus $25.2 \%$, OR 0.52 , IC $95 \% 0.29$ $0.96, \mathrm{p}=0.03$ ). Las diferencias en mortalidad continuaron siendo significativas en pacientes con compromiso pulmonar severo (38.8\% vs 80.7\%, OR 0.15, IC 95\% 0.05- 0.47, $\mathrm{p}=0.001$ ). De igual manera, después del ajuste de posibles variables confusoras a través de un análisis multivariado, las diferencias en la mortalidad fueron significativas (OR 0.27 , IC $95 \%$ 0.09-0.80, p=0.03) (20). En la metodología del artículo se menciona adicionalmente un análisis secundario mediante un pareamiento por puntaje de propensiones (propensity score) corroborando los hallazgos del análisis multivariado.

Como se ve en el ejemplo, se trata de un estudio de seguimiento donde se obtienen medidas de asociación, intervalos de confianza que no atraviesan la hipótesis nula, valores de $\mathrm{p}$ menores a 0.05 y donde además se han utilizado herramientas estadísticas como la regresión multivariada o el propensity score para limitar la posibilidad de confusión y brindar mayor solidez a los resultados del estudio. Resultados de este estudio han sido ampliamente contrastados con muchos otros de pobre calidad metodológica y éticamente cuestionados.

\section{RESPUESTA CLÍNICAMENTE FAVORABLE}

Frecuentemente y en particular en el ámbito médico, existe la costumbre de hacer una valoración bastante simplista de las medidas de asociación catalogando su relevancia en virtud del valor de "p". Así, casi de manera inmediata un valor de p< 0.05 se postula como la conclusión más relevante de un manuscrito sin considerar las implicaciones y la información por demás bastante ilustrativa que ofrecen la interpretación de los intervalos de confianza. Se sugiere que dicha interpretación se haga en virtud de un efecto o respuesta clínicamente favorable. Por ejemplo, y para resaltar el impacto de las medidas de asociación más notorias del estudio de Rajter, retomemos la reducción de la mortalidad (15\% versus $25.2 \%$, con un OR 0.52, IC 95\% 0.29-0.96, p=0.03). Sin embargo, el estudio es observacional analítico y es mejor esperar resultados en ensayos clínicos controlados para tomar una mejor decisión frente a los pacientes.

Por otra parte, debe tenerse en cuenta que el valor de $\mathrm{p}$, a pesar de su sobrevalorada popularidad tiene importantes limitaciones puesto que, no brinda información relacionada con la magnitud o dirección de diferencias evaluadas, razón por la cual su interpretación deberá siempre analizarse en el marco de los intervalos de confianza.

\section{PERFIL FARMACOLÓGICO SEGURO}

Este criterio supone que el medicamento a emplear no esté contraindicado y no represente un riesgo significativo para la salud del paciente. En este punto se debe ponderar cuidadosamente la relación riesgo-beneficio manteniendo como criterio orientador la premisa Primun non nocere. El empleo de medicamentos off-label podría parecer una alternativa práctica dado que su perfil de seguridad ya ha sido probado en otras condiciones médicas, pero el empleo de un nuevo fármaco debe atravesar por distintas fases preclínicas y clínicas para demostrar que se trata de un medicamento seguro y este proceso puede tardar varios ańos.

La ivermectina es uno de los pocos ejemplos en la farmacología donde se ha demostrado un perfil de seguridad bastante amplio. Se trata de un medicamento derivado de las avermectinas, aisladas de la fermentación del Streptomyces avermectinius y compuesta de una mezcla que contiene $80-90 \%$ de 22.23 -dihidroavermectina B1a y $10-20 \%$ de 22.23-dihidroavermectina B1b (21).

Se han descrito algunas reacciones adversas en tratamientos contra filariasis y no se recomienda emplearlo en nińos 
menores de 2 ańos o con menos de 15 kilos de peso dado que puede atravesar la barrera hematoencefálica todavía inmadura en esta edad (21). De manera excepcional se ha documentado cuadros de disfunción neurológica, ataxia y coma presuntamente relacionadas con mutaciones en una proteína transportadora denominada mdr-1 (22). No obstante, en general la ivermectina es un medicamento bien tolerado dado que se concentra esencialmente en el tejido adiposo y tiene muy poca afinidad por el Sistema Nervioso Central lo cual le brinda un perfil de seguridad bastante amplio (23).

El uso global del medicamento durante más de 40 ańos para el tratamiento de parasitosis como la oncocercosis y la filariasis $(24,25)$ con mínimas reacciones adversas, hacen del medicamento una opción bastante segura en comparación a otras alternativas off-label frente al COVID. El empleo masivo de la ivermectina en programas internacionales en África y Latinoamérica demuestra la seguridad del medicamento (21).

Algunos autores han planteado dudas acerca de la dosis efectiva de ivermectina frente al SARS-CoV-2 $(26,27)$. Esencialmente se ha planteado que para lograr un efecto farmacológico similar al del estudio in vitro realizado por Caly y colaboradores (12), serían necesarias dosis excesivamente elevadas que pudiesen ser tóxicas (28). Sin embargo, tal como lo menciona el autor al responder a estas inquietudes, inclusive dosis pequeńas de ivermectina tendrían la posibilidad de modulares procesos inmunológicos frente al SARS-CoV-2 y reducir, aunque sea de manera discreta la carga viral. Estos efectos le darían al paciente la posibilidad de controlar y responder mejor frente a la infección viral (28). En el mismo sentido, los hallazgos documentados por Rajter y colaboradores muestran que una dosis de 200 microgramos/kilo -dosis considerada segura- parece ser efectiva desde el punto de vista clínico, brindando respaldo a la respuesta dada por Caly frente a estas preocupaciones (28).

\section{FACTIBILIDAD E INTERÉS ALTRUISTA}

El listado de terapias en estudio contra el COVID es bastante amplio e incluye medicamentos de uso relativamente común como la ivermectina, cloroquina, la azitromicina, los corticosteroides y otros de uso inusual, no disponibles o muy costosos como el tocilizumab, remdesivir, interferon, siltuximab, favipiravir, ácido lipoico o plasma convaleciente por poner solo algunos ejemplos (29-32).

Naturalmente, entre los medicamentos disponibles es necesario considerar la factibilidad del uso masivo en el contexto de una pandemia. A este respecto cabe igualmente un análisis de los costos de los tratamientos y si los sistemas de salud y los gobiernos son capaces de asegurar el acceso de toda la población a las terapias. Del mismo modo, resulta conveniente incluir en este análisis losintereses económicos del sector farmacéutico, de organizaciones científicas y de algunos gobiernos por privilegiar la investigación de terapias específicas sin ponderar el criterio científico y el valor altruista de la investigación. En este sentido, resultan inquietantes por ejemplo, los costos de un posible tratamiento con remdesivir, que pueden superar los 3100 dólares, así como los movimientos bursátiles de algunas companías farmacéuticas $(33,34)$. Desafortunadamente, en la historia de la medicina son múltiples los ejemplos donde se han antepuesto los intereses económicos sobre el interés de ayudar a los pacientes. La débil investigación e inversión en terapias contra enfermedades olvidadas de Latinoamérica y de África, las cuales afectan a miles de personas pero que no representan ganancias monetarias, contrastan diametralmente con las investigaciones y la inversión en tratamientos para enfermedades huérfanas de pacientes Europeos o de Estados Unidos mucho más rentables económicamente (35).

La disponibilidad de la ivermectina, su bajo costo y el genuino interés de servir que debe caracterizar al médico y al investigador, son elementos que harían de este medicamento una alternativa, en particular en países de América Latina. Al igual que en otras terapias, se esperan ensayos clínicos bien realizados, conducidos éticamente y robustos para tomar decisiones apropiadas y bien informadas.

\section{CONCLUSIONES}

Antes que proponer una terapia específica, el objetivo de este manuscrito es poner en consideración de la comunidad médica y científica una serie de criterios a la hora de ponderar una hipótesis de investigación y el empleo de un medicamento off-label frente al COVID-19. Aspectos como la plausibilidad biológica, evidencia metodológicamente aceptable, evidencia de un efecto clínicamente importante, un perfil farmacológico seguro, factibilidad e interés altruista deben respaldar la puesta en marcha de protocolos de investigación y el uso compasivo de terapias off-label. Aunque a la luz de los criterios mencionados la ivermectina pareciera una buena alternativa frente al COVID, su efectividad debe evaluarse en un ambiente hospitalario, o de forma ambulatoria con acompańamiento y vigilancia médica, preferiblemente con protocolos de investigación para uso de medicamentos off-label o en el contexto de 
ensayos clínicos bien diseńados. En este sentido, debe resaltarse que, hasta el momento, parte de la información relacionada con la ivermectina frente a la COVID proviene de estudios con riesgo de sesgos y el nivel de evidencia clínico es muy bajo. Mas recientemente, varios estudios de ivermectina han sido cuestionados y retractados por cuestiones éticas mayores durante su conducción.

Como las investigaciones terapéuticas en la actual pandemia avanzan a buen ritmo, hallazgos de recientes investigaciones que incluyen ensayos clínicos y metaanálisis han demostrado que la ivermectina carece de un verdadero efecto frente a la Covid-19. El estudio de López y colaboradores mostró por ejemplo que la ivermectina no mejora significativamente el tiempo de resolución de los síntomas comparados con placebo (36). Del mismo modo, una revisión sistemática recientemente publicada en la revista Clinical Infectious Disease que recoge la información de 10 ensayos clínicos y más de 1000 pacientes, mostró que la ivermectina no redujo la mortalidad o el tiempo de aclaramiento viral cuando se compara con el placebo o el cuidado médico estándar (37).

Por esta razón, se enfatiza que el objeto de este manuscrito no es el de promover una terapia específica frente a la enfermedad, sino el de evaluar distintos criterios a la hora de priorizar un medicamento off-label para uso compasivo o para el desarrollo de protocolos de investigación que generen evidencia robusta.

Seinvitaalacomunidad científicaymédica especialmentede Latinoamérica a evaluar la efectividad de los medicamentos analizando de manera crítica la relación riesgo/beneficio, la literatura médica disponible y considerando los criterios arriba descritos. Se debe alentar igualmente el uso de este tipo de terapias en el contexto de protocolos de investigación bien diseńados. Solo de esta manera se puede generar evidencia de buena calidad fortaleciendo además la confianza en el método científico como una herramienta válida y útil para resolver los problemas de salud.

\section{CONTRIBUCIÓN DE LOS AUTORES}

El autor se encargó de la concepción de la idea, diseńo del manuscrito, análisis de la información, redacción del artículo, correcciones y aprobación final del manuscrito.

\section{CONFLICTOS DE INTERÉS}

el autor del manuscrito declara no tener ningún conflicto de interés alguno

\section{FUENTES DE FINANCIAMIENTO}

Ninguna

\section{REFERENCIAS}

1. Kupferschmidt K, Cohen J. Race to find COVID-19 treatments accelerates. Science 2020; 367:1412-3. Doi: 10.1126/science.367.6485.1412

2. WHO, UN Foundation and partners launch first-of-its-kind COVID-19 Solidarity Response Fund [Internet]. World Healt Organization. 2020 [Citado: mayo 06, 2020]. Disponible en: https://www.who.int/news/item/13-03-2020-who-unfoundation-and-partners-launch-first-of-its-kind-covid-19solidarity-response-fund

3. Launch of a European clinical trial against COVID-19 [Internet]. Institut national de la santé et de la recherche médicale. 2020. [Citado: mayo 06, 2021]. Disponible en: https://presse.inserm.fr/en/launch-ofa-european-clinical-trial-against-covid-19/38737/

4. Kalil AC. Treating COVID-19-Off-Label Drug Use, Compassionate Use, and Randomized Clinical Trials During Pandemics. JAMA. 2020; 323(19):1897-8. doi:10.1001/jama.2020.4742

5. Ongoing Living Update of Potential COVID-19 Therapeutics: summary of rapid systematic reviews [Internet]. Pan American Health Organization. 2020. [Citado: mayo 08, 2020]. Disponible en: https://iris.paho.org/bitstream/ handle/10665.2/52025/PAHOIMSCovid19200008_eng. pdf?sequence $=1 \&$ isallowed $=y$

6. Mehra MR, Desai SS, Ruschitzka F, Patel AN. RETRACTED: Hydroxychloroquine or chloroquine with or without a macrolide for treatment of COVID-19: a multinational registry analysis. The Lancet. 2020: S0140-6736(20)31180-6. https://doi.org/10.1016/ S0140-6736(20)31180-6

7. Paumgartten FJR, Oliveira ACAX de, Paumgartten FJR, Oliveira ACAX de. Off label, compassionate and irrational use of medicines in Covid-19 pandemic, health consequences and ethical issues. Cięnc Amp Saúde Coletiva. 2020; 25(9):3413-9. https://doi. org/10.1590/1413-81232020259.16792020 
8. WHO COVID-19 Solidarity Therapeutics Trial [Internet]. World Healt Organization. 2020. [Citado: octubre 27, 2020]. Disponible en: https://www.who. int/emergencies/diseases/novel-coronavirus-2019/ global-research-on-novel-coronavirus-2019-ncov/ solidarity-clinical-trial-for-covid-19-treatments

9. Manterola DC, Zavando MD. Cómo interpretar los "Niveles de Evidencia" en los diferentes escenarios clínicos. Rev Chil Cir. 2009; 61:582-95. https://doi. org/10.4067/S0718-40262009000600017

10. Tay MYF, Fraser JE, Chan WKK, Moreland NJ, Rathore AP, Wang C, et al. Nuclear localization of dengue virus (DENV) 1-4 non-structural protein 5; protection against all 4 DENV serotypes by the inhibitor Ivermectin. Antiviral Res. 2013;99:301-6. https://doi.org/10.1016/j. antiviral.2013.06.002

11. Varghese FS, Kaukinen P, Gläsker S, Bespalov M, Hanski L, Wennerberg K, et al. Discovery of berberine, abamectin and ivermectin as antivirals against chikungunya and other alphaviruses. Antiviral Res. 2016; 126:117-24. https://doi. org/10.1016/j.antiviral.2015.12.012

12. Caly L, Druce JD, Catton MG, Jans DA, Wagstaff KM. The FDA-approved drug ivermectin inhibits the replication of SARS-CoV-2 in vitro. Antiviral Res. 2020; $178: 104787$. https://doi.org/10.1016/j.antiviral.2020.104787

13. Petrosillo N, Viceconte G, Ergonul O, Ippolito G, Petersen E. COVID-19, SARS and MERS: are they closely related?. Clin Microbiol Infect. 2020; 26:729-34. https://doi.org/10.1016/j.cmi.2020.03.026

14. Frieman M, Yount B, Heise M, Kopecky-Bromberg SA, Palese P, Baric RS. Severe Acute Respiratory Syndrome Coronavirus ORF6 Antagonizes STAT1 Function by Sequestering Nuclear Import Factors on the Rough Endoplasmic Reticulum/ Golgi Membrane. J Virol. 2007; 81:9812-24. https://doi. org/10.1128/JVI.01012-07

15. Billiau A. Interferon-gamma: biology and role in pathogenesis. Adv Immunol. 1996; 62:61-130. https:// doi.org/10.1016/s0065-2776(08)60428-9

16. Kindler E, Thiel V. SARS-CoV and IFN: Too Little, Too Late. Cell Host Microbe. 2016; 19:139-41. https://doi. org/10.1016/j.chom.2016.01.012

17. Hernández-Avila M, Garrido-Latorre F, López-Moreno S. Diseńo de estudios epidemiológicos. Salud Pública México. 2000; 42:144-54. https://doi.org/10.1590/ S0036-36342000000200010

18. Veiga de Cabo J, Fuente Díez E de la, Zimmermann Verdejo M. Modelos de estudios en investigación aplicada: conceptos y criterios para el diseńo. Med Segur Trab. 2008; 54:81-8
19. Silva-Aycaguer LC. Las pruebas de significación estadística: seis décadas de fuegos artificiales. Rev Fac Nac Salud Pública. 2016; 34 (3):372-9. https://doi. org/10.17533/udea.rfnsp.v34n3a11

20. Rajter JC, Sherman MS, Fatteh N, Vogel F, Sacks J, Rajter J-J. Use of Ivermectin Is Associated With Lower Mortality in Hospitalized Patients With Coronavirus Disease 2019. Chest Journal. 2020; 159(1):85-92. https://doi.org/10.1016/j. chest.2020.10.009

21. Ch JV. Ivermectina: Sus Múltiples Usos, Seguridad y Toxicidad. Educación Médica Continua. 2010; 26(4):358-368

22. Chandler RE. Serious Neurological Adverse Events after Ivermectin-Do They Occur beyond the Indication of Onchocerciasis?. Am J Trop Med Hyg. 2018; 98(2):3828. https://doi.org/10.4269/ajtmh.17-0042

23. Lespine A, Dupuy J, Alvinerie M, Comera C, Nagy T, Krajcsi P, et al. Interaction of macrocyclic lactones with the multidrug transporters: the bases of the pharmacokinetics of lipidlike drugs. Curr Drug Metab. 2009; 10:272-88. https:// doi.org/10.2174/138920009787846297

24. Kyelem D, Biswas G, Bockarie MJ, Bradley MH, El-Setouhy $\mathrm{M}$, Fischer PU, et al. Determinants of success in national programs to eliminate lymphatic filariasis: a perspective identifying essential elements and research needs. Am J Trop Med Hyg. 2008; 79:480-4

25. Osei-Atweneboana MY, Eng JK, Boakye DA, GyapongJO, Prichard RK. Prevalence and intensity of Onchocerca volvulus infection and efficacy of ivermectin in endemic communities in Ghana: a two-phase epidemiological study. The Lancet. 2007; 369(9578):2021-9. https:// doi.org/10.1016/S0140-6736(07)60942-8

26. Momekov G, Momekova D. Ivermectin as a potential COVID-19 treatment from the pharmacokinetic point of view: antiviral levels are not likely attainable with known dosing regimens. MedRxiv. 2020.04.11.20061804. https:// doi.org/10.1101/2020.04.11.20061804

27. Schmith VD, Zhou J, Lohmer LR. The Approved Dose of Ivermectin Alone is not the Ideal Dose for the Treatment of COVID-19. Clin Pharmacol Ther. 2020; 108(4):762-765. https://doi.org/10.1002/cpt.1889

28. Bray M, Rayner C, Noël F, Jans D, Wagstaff K. Ivermectin and COVID-19: A report in Antiviral Research, widespread interest, an FDA warning, two letters to the editor and the authors' responses. Antiviral Res. 2020; 178:104805. https://doi.org/10.1016/j.antiviral.2020.104805

29. McCreary EK, Pogue JM. Coronavirus Disease 2019 Treatment: A Review of Early and Emerging Options. Open Forum Infect Dis. 2020; 7(4):ofaa105. https:// doi.org/10.1093/ofid/ofaa105 
30. Zhong M, Sun A, Xiao T, Yao G, Sang L, Zheng X, et al. A Randomized, Single-blind, Group sequential, Activecontrolled Study to evaluate the clinical efficacy and safety of $\alpha$-Lipoic acid for critically ill patients with coronavirus disease 2019(COVID-19). MedRxiv. 2020:04.15.20066266. https://doi.org/10.1101/2020.04.15.20066266

31. Gritti G, Raimondi F, Ripamonti D, Riva I, Landi F, Alborghetti L, et al. Use of siltuximab in patients with COVID-19 pneumonia requiring ventilatory support. MedRxiv. 2020: 04.01.20048561. https://doi.org/10.11 01/2020.04.01.20048561

32. Sanders JM, Monogue ML, Jodlowski TZ, Cutrell JB. Pharmacologic Treatments for Coronavirus Disease 2019 (COVID-19): A Review. JAMA. 2020; 323(18):182436. https://doi.org/10.1001/jama.2020.6019

33. Ahmed S, Johnson K. Operaciones bursátiles en Gilead por fármaco contra COVID-19 podrían llamar atención de reguladores [Internet]. Reuters. 2020. [Citado: noviembre 02, 2021]. Disponible en: https:// www.reuters.com/article/salud-coronavirus-gileadidLTAKBN2382VS

34. Lupkin S. Remdesivir Priced At More Than $\$ 3100$ For A Course of Treatment [Internet]. National Public Radio. 2020. [Citado: octubre 27, 2020). Disponible en: https://www.npr.org/sections/healthshots/2020/06/29/884648842/remdesivir-priced-atmore-than-3-100-for-a-course-of-treatment

35. Marina AM, Jaime KJ, Consuelo MB, Francisco LN, Manuel MNJ. Las enfermedades "olvidadas" de América Latina y el Caribe: un problema de salud pública global. Revista mexicana de ciencias farmacéuticas. 2012; 43(1):33-41

36. López-Medina E, López P, Hurtado IC, Dávalos DM, Ramirez 0, Martínez E, et al. Effect of Ivermectin on Time to Resolution of Symptoms Among Adults With Mild COVID-19 A Randomized Clinical Trial. JAMA. 2021;325(14):1426-1435. https://doi.org/10.1001/ jama.2021.3071

37. Roman YM, Burela PA, Pasupuleti V, Piscoya A, Vidal JE, Hernandez AV. Ivermectin for the Treatment of Coronavirus Disease 2019: A Systematic Review and Meta-analysis of Randomized Controlled Trials. Clin Infect Dis. 2021. https://doi.org/10.1093/cid/ciab591 